\title{
SWELLING-INDUCED INSTABILITIES OF POLYMERIC HYDROGELS WITH PERIODIC MICROSTRUCTURES
}

\author{
ELTEN POLUKHOV ${ }^{1}$ AND MARC-ANDRÉ KEIP ${ }^{1}$ \\ ${ }^{1}$ Institute of Applied Mechanics, Department of Civil and Environmental Engineering, \\ University of Stuttgart \\ Pfaffenwaldring 7, 70569 Stuttgart, Germany \\ \{elten.polukhov;marc-andre.keip\}@mechbau.uni-stuttgart.de,www.mib.uni-stuttgart.de
}

Key words: Hydrogels, Stability Analysis, Pattern Transformations, Buckling, Transient Problems

\begin{abstract}
Hydrogels are soft, hydrophilic materials which can absorb a large volume of solvent and undergo finite volumetric deformations known as swelling. The swelling of a hydrogel can be a driving mechanism for complex material responses such as pattern transformation which lead to change of periodicity as a result of a microscopic instability in periodic materials. In the present contribution, we deal with the computational analysis of swelling-induced instabilities in periodic hydrogels. The stability analysis based on the Bloch-Floquet theory is carried out within a transient two-field minimization-type variational principle. The presented formulation and methodology for the stability analysis are computationally efficient, since the computations are carried out on the smallest representative volume element of the microstructure. Within this framework, we study swelling-induced microscopic instabilities for various perforated hydrogels. Our findings are consistent with experimental observations and show that the so-called diamond plate patterns are the critical buckling mode for voided microstructures. Moreover, we observe long-wavelength instabilities for certain volume fractions of voids.
\end{abstract}

\section{INTRODUCTION}

Hydrogels are soft, porous, hydrophilic, biocompatible materials having a polymer network structure which is retained together as a result of existence of physical and chemical crosslinks. The polymer network structure gives hydrogels favorable elastic behavior. Hydrogels can absorb a large volume of water into the network of polymer chains; as a result they undergo finite volumetric deformation referred to as swelling [1]. Since the swelling of hydrogels can be also controlled by various external stimuli in addition to their favorable properties, these soft materials have a wide range of applications, e.g., in the development of micro and biomedical devices [2, 3, 4]. Similarly, other important application areas are realized by use of periodic hydrogels, where the specially designed microstructures allow obtaining further controllable patterns and tunable responses. Here, we refer to metamaterials with negative overall volume change under the diffusion of solvent into the system $[5,6]$.

Diffusion-deformation processes in hydrogels involve interaction of complex elastic and chemical processes. This interaction can be a driving mechanism for further complex responses such as development of instabilities and pattern transformations in soft hydrogels [7,8]. Understanding the instabilities in the soft materials can also have important implications for the growth of biological systems, refer to [9] for 
a review of mechanical instabilities in gels and references therein.

In the present contribution, we are interested in the investigation of instabilities, particularly pattern transforming microscopic instabilities in periodic hydrogels $[8,10]$. The instability analysis of equilibrium swelling in periodic structures has been already considered in [11, 12, 5]. However, these references do not account for the transient diffusion phenomenon. In the present contribution, our aim is to introduce a methodology to study the pattern-transforming instabilities in periodic hydrogels based on Bloch-Floquet wave analysis within a transient variational framework. This will allow to detect the critical instabilities on a chosen smallest representative domain of hydrogels which we refer to as unit-cell representative volume element (RVE). Although the computations will be on unit-cell RVE, the framework allows to detect the critical size of periodic units of a hydrogel which can go beyond the unit-cell size. This makes the numerical stability analysis also computationally efficient.

Our stability analysis is embedded into a minimization-type variational framework which allows to consider the stability analysis in the classical energy-based sense. Additionally, the minimization nature of the formulation has also favorable implications such as positive-definiteness until instability point and symmetry of underlying system of equations $[13,14,16]$. Although this formulation is favorable to investigate instabilities and it is not constrained by the inf-sup condition, it also requires a discretization using special elements such as Raviart-Thomas-type finite elements [17, 18].

In the following, we first start with the rate-type variational formulation of dissipative deformationdiffussion processes and its implementation into a time-space discrete numerical framework. Within the numerical framework, we consider the implementation of Bloch-Floquet-type stability analysis. Fi-

nally, we consider numerical examples based on experiments and demonstrate the capabilities of the
framework. The numerically obtained pattern fornation is in line with experinental observations. We
conclude the contribution with summary and an outlook.
2 VARIATIONAL FORMULATION OF DIFHUSION-DEFORMATION PROCESSES

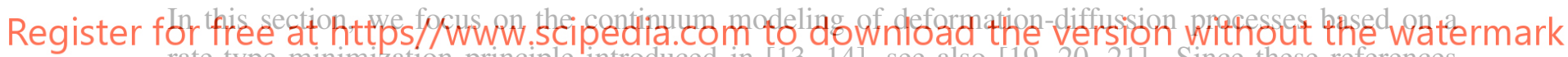
rate-type minimization principle introduced in $[13,14]$, see also $[19,20,21]$. Since these references provide a detailed description of the formulation, in the following, our presentation is given in a concise form. Within our formulation, we also account for time-space discretization of the problem, whereby we consider the incremental stability analysis of periodic hydrogel microstructures.

\subsection{Independent primary fields of the minimization formulation}

Since we consider a minimization-type formulation, the constitutive state of a deformation-diffusion phenomenon can be described by the deformation map $\varphi$ and solvent-volume flux vector $\mathbb{H}$

$$
\boldsymbol{\varphi}:\left\{\begin{array}{l}
\mathcal{B}_{0} \times \mathcal{T} \rightarrow \mathcal{B}_{t} \subset \mathcal{R}^{3} \\
(\mathbf{X}, t) \mapsto \boldsymbol{\varphi}(\mathbf{X}, t)
\end{array} \quad \text { and } \quad \mathbb{H}:\left\{\begin{array}{l}
\mathcal{B}_{0} \times \mathcal{T} \rightarrow \mathcal{B}_{t} \subset \mathcal{R}^{3} \\
(\mathbf{X}, t) \mapsto \mathbb{H}(\mathbf{X}, t)
\end{array}\right.\right.
$$

where $\mathcal{B}_{0}$ and $\mathcal{B}_{t}$ denote the reference and current configuration of a porous material, respectively. While the deformation map determines the position of the material points $\mathbf{X}$ of the whole mixture $\mathcal{B}_{0}$ in the current configuration $\mathcal{B}_{t}$ at time $t \subset \mathcal{T}$, the Lagrangian solvent-volume flux vector $\mathbb{H}$ describes the relative 


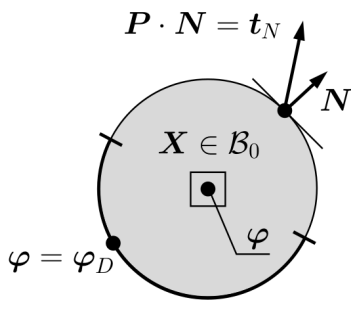

deformation field

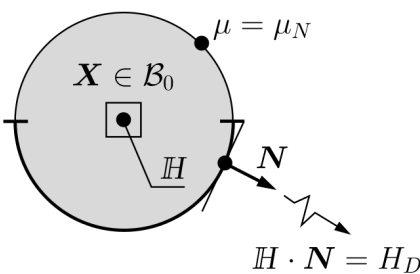

fluid-volume flux field

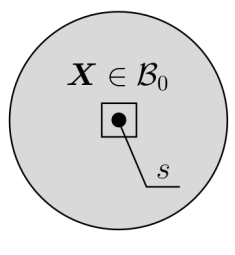

swelling field

Figure 1: Description of global primary fields with corresponding boundary conditions and a local field without a boundary condition of the two-field minimization formulation.

volume transport rate of a solvent across the area element $\mathrm{d} \mathbf{A}$ of an infinitesimal part $\mathfrak{P}_{0} \subset \mathcal{B}_{0}$ w.r.t. the motion of the mixture, refer to $[22,23]$ for details. Although we consider a Lagrangian formalism in the present work, the Eulerian solvent-volume flux can be related to the Lagrangian field via

$$
\mathbb{h} \cdot \mathrm{d} \mathbf{a}=\mathbb{H} \cdot \mathrm{d} \mathbf{A} \quad \Rightarrow \quad \mathbb{H}=J \mathbf{F}^{-T} \mathbb{h} .
$$

In addition to the above global fields, we also introduce the solvent-volume concentration $s$ over an infinitesimal part $\mathcal{P}_{0} \subset \mathcal{B}_{0}$, see Fig. 1. The solvent-volume concentration is related to the volume-flux

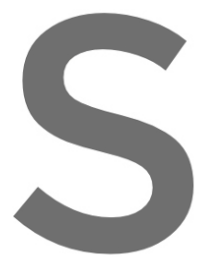
vector via Gauss' law

The equation (3) is also introduce the deformation
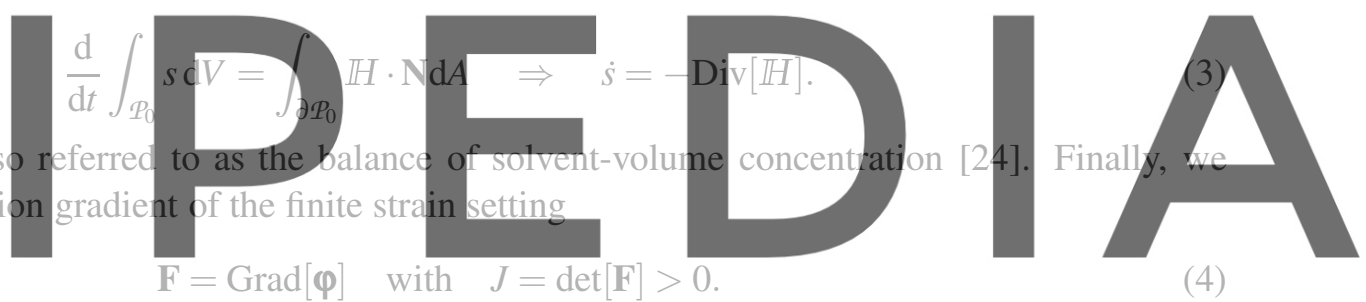

(4)

Register for free at https//www.scipedia.com to download the version without the watermark 2.2 Constitutive functions of deformation-diffusion process

The second axiom of thermodynamics requires that the evolution of the stored energy within a part $\mathcal{P}_{0}$ does not exceed the external power acting on this part

$$
\frac{\mathrm{d}}{\mathrm{d} t} \int_{\mathcal{P}_{0}} \psi \mathrm{d} V \leq \underbrace{\int_{\partial \mathcal{P}_{0}} \mathbf{t} \cdot \dot{\varphi} \mathrm{d} A-\int_{\partial \mathcal{P}_{0}} \mu \mathbb{H} \cdot \mathbf{N} \mathrm{d} A}_{P_{\text {ext }}\left(\mathcal{P}_{0}\right)},
$$

where $\mathbf{t}=\mathbf{P} \cdot \mathbf{N}$ is the nominal traction vector acting on the part $\mathcal{P}_{0}$ due to the action of the rest of the body $\mathcal{B}_{0} \backslash \mathcal{P}_{0} ; \mu$ is the chemical potential of the solvent describing the energy conducted to the part $\mathcal{P}_{0}$ as a result of solvent-volume influx.

From the second axiom of thermodynamics (5), we can obtain the dissipation associated with the part $\mathcal{P}_{0}$ as the difference between the power of external loading and the evolution of the stored energy

$$
\int_{\mathcal{P}_{0}} \mathcal{D} \mathrm{d} V:=P_{\text {ext }}\left(\mathcal{P}_{0}\right)-\frac{\mathrm{d}}{\mathrm{d} t} \int_{\mathcal{P}_{0}} \psi \mathrm{d} V \geq 0 .
$$


By localizing the latter inequality, we obtain the so-called local and conductive parts of the localized dissipation inequality [24], which need to be satisfied separately

$$
\mathcal{D}_{l o c}:=\mathbf{P}: \dot{\mathbf{F}}+\mu \dot{s}-\dot{\psi} \geq 0 \quad \text { and } \quad \mathcal{D}_{\text {dif }}:=\mathbb{H} \cdot \mathbb{M} \geq 0,
$$

where $\mathbf{P}$ is the first Piola-Kirchhoff stress tensor; $\mathbb{M}=-\mathrm{Grad} \mu$ is the driving force of the diffusion process. Since we assume that the relaxation time of the material is much lower than the characteristic diffusion time of the solvent, we do not take into account any viscoelastic response of the skeleton due to the diffusion of the solvent. Consequently, the dissipation within the current formulation can be associated merely to the diffusion process.

From (7) $)_{1}$, we define the energy-storage density function $\hat{\psi}(\mathbf{F}, s)$ per unit volume of the reference configuration and associated energy-storage functional $E(\mathbf{F}, s)$ of the body $\mathcal{B}_{0}$ in the following forms

$$
\hat{\psi}(\mathbf{F}, s):=\hat{\psi}_{\text {mech }}(\mathbf{F})+\hat{\psi}_{\text {chem }}(s)+\hat{\psi}_{\text {coup }}(J, s) \Rightarrow E(\mathbf{F}, s):=\int_{\mathcal{B}_{0}} \hat{\psi}(\mathbf{F}, s) \mathrm{d} V,
$$

which we take into account in $(7)_{1}$. As a result, we determine the first Piola-Kirchhoff stress tensor $\mathbf{P}$ and chemical potential $\mu$ constitutively from the energy-storage density function $\hat{\psi}(\mathbf{F}, s)$

$$
\mathbf{P}=\partial_{\mathbf{F}} \hat{\psi}(\mathbf{F}, s) \quad \text { and } \quad \mu=\partial_{s} \hat{\psi}(\mathbf{F}, s) .
$$

The inequality $(7)_{2}$ is satisfied a priori by introducing a positive, normalized and convex dissipation density function $\hat{\phi}(\mathbb{H})$ per of the body $\mathcal{B}_{0}$ in the follo
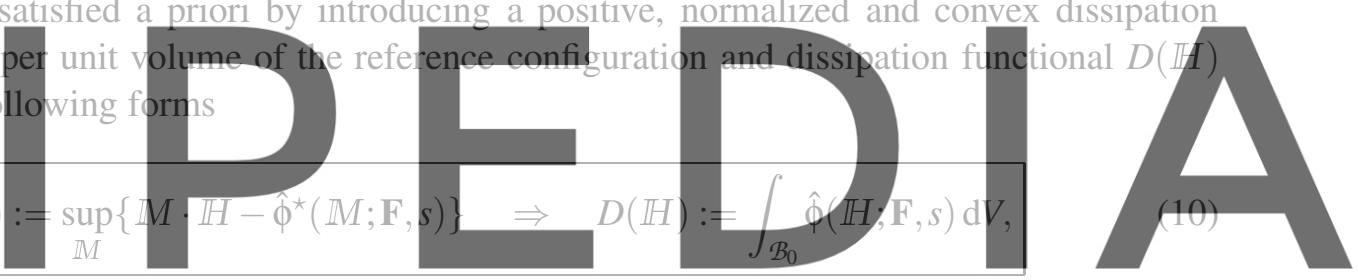
Register for fre $\hat{\phi}^{\star}$ (Mt hist the dual dissipation density function. As a resultwe determine the diffusion-driving
orce

$$
\mathbb{M} N=\partial_{\mathbb{H}} \hat{\phi}(\mathbb{H I} ; \mathbf{F}, s) .
$$

Both energy-storage and dissipation density functions will be given explicitly in Section 4 .

\subsection{Rate-type two-field minimization principle}

Based on the above defined functionals in $(8)_{2}$ and $(10)_{2}$, we can postulate the rate-type potential functional in the following form $[13,14,15]$

$$
\Pi(\dot{\boldsymbol{\varphi}}, \mathbb{H}):=\frac{\mathrm{d}}{\mathrm{d} t} E(\dot{\boldsymbol{\varphi}}, \mathbb{H})-P_{e x t}(\dot{\boldsymbol{\varphi}}, \mathbb{H})=\int_{\mathcal{B}_{0}} \pi(\dot{\boldsymbol{\varphi}}, \mathbb{H}) \mathrm{d} V-P_{e x t}(\dot{\boldsymbol{\varphi}}, \mathbb{H})
$$

where $\pi(\dot{\varphi}, \mathbb{H})$ is the rate-type potential density of the solid-fluid mixture and defined as follows

$$
\pi(\dot{\boldsymbol{\varphi}}, \mathbb{H}):=\frac{\mathrm{d}}{\mathrm{d} t} \hat{\psi}(\mathbf{F}, s)+\hat{\phi}(\mathbb{H} ; \mathbf{F}, s)=\partial_{\mathbf{F}} \hat{\psi}(\mathbf{F}, s): \dot{\mathbf{F}}-\partial_{s} \hat{\psi}(\mathbf{F}, s) \operatorname{Div}[H]+\hat{\phi}(\mathbb{H} ; \mathbf{F}, s),
$$


where we have used the balance of solvent-volume concentration $\dot{s}=-\operatorname{Div}[H]$. The external power applied to the body $\mathcal{B}_{0}$ is determined in the following form

$$
P_{e x t}(\dot{\boldsymbol{\varphi}}, \mathbb{H}):=\int_{\partial \mathcal{B}_{0}} \mathbf{t} \cdot \dot{\varphi} \mathrm{d} A-\int_{\partial \mathcal{B}_{0}} \mu \mathbb{H} \cdot \mathbf{N} \mathrm{d} A
$$

Based on the potential functional (12), we state the minimization principle as follows

$$
\left\{\dot{\varphi}^{\star}, \mathbb{H}^{\star}\right\}=\operatorname{Arg}\left\{\inf _{\dot{\varphi} \in \mathcal{W}_{\varphi}} \inf _{\mathbb{H} \in \mathcal{W}_{H}} \Pi(\dot{\boldsymbol{\varphi}}, \mathbb{H})\right\}
$$

where the admissible spaces for the rate of deformation and the solvent-volume flux vector are

$$
\begin{aligned}
\mathcal{W}_{\dot{\varphi}} & :=\left\{\dot{\boldsymbol{\varphi}} \in H^{1}\left(\mathcal{B}_{0}\right) \mid \dot{\boldsymbol{\varphi}}=\dot{\boldsymbol{\varphi}}_{D} \text { on } \mathcal{B}_{0}^{\dot{\varphi}}\right\} \\
\mathcal{W}_{\mathbb{H}} & :=\left\{\mathbb{I} I \in H\left(\operatorname{Div}, \mathcal{B}_{0}\right) \mid \mathbb{H} \cdot \mathbf{N}=H_{D} \text { on } \mathcal{B}_{0}^{H}\right\}
\end{aligned}
$$

Considering the necessary condition of the variational principle at equilibrium, we obtain the EulerLagrange equations

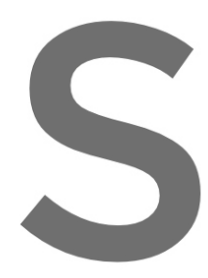

$\boldsymbol{S}^{2}$

2.4 Time-Space discret

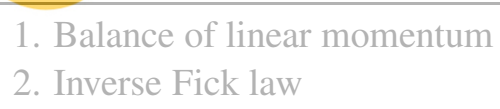

2. Inverse Fick law

3. Traction boundary condition

To implement the above variational formulation into a confor

$$
\begin{aligned}
& \operatorname{Div}\left[\partial_{\mathbf{F}} \hat{\psi}\right]=0 \\
& \operatorname{Grad}\left[\partial_{S} \hat{\psi}\right]+\partial_{\mathbb{H}} \hat{\phi}=0 \\
& \partial_{\mathbf{F}} \hat{\psi} \cdot \mathbf{N}-\mathfrak{t}=\mathbf{0}
\end{aligned}
$$

consider a time discretization using an implicit Euler scheme in a time interval $\tau=t_{n+1}-t_{n}$. As a result,

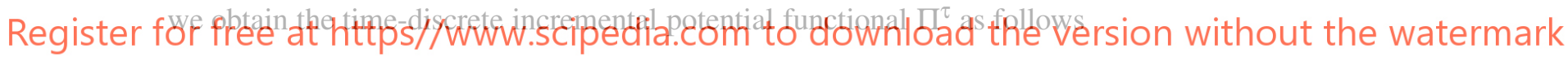

$$
\Pi^{\tau}(\dot{\varphi}, \mathbb{H}):=\int_{\mathcal{B}_{0}} \hat{\psi}\left(\mathbb{F}, s_{n}-\tau \operatorname{Div}[\mathbb{H}]\right)+\tau \hat{\phi}\left(\mathbb{H} ; \mathbb{F}_{n}, s_{n}\right) \mathrm{d} V-\tau P_{\text {ext }}(\dot{\varphi}, \mathbb{H}),
$$

where we drop the subscript for the variables at the current time step. Additionally, we determine the solvent-volume concentration at time $t_{n+1}$ via the discrete version of the balance of solvent-volume concentration, i.e., $s_{n+1}=s_{n}-\tau \operatorname{Div}[H]$. The admissible spaces for the deformation map and solvent-volume flux are the discrete versions of (16). In the present contribution, we use a Raviart-Thomas-type $\mathrm{Q}_{2}-\mathrm{RT}_{0}$ finite-element formulation for the space discretization in line with the requirements of the admissible spaces $[17,18]$. Consequently, the discrete incremental version of the variational principle (15) is given as

$$
\boldsymbol{d}^{\star}=\operatorname{Arg}\left\{\min _{\boldsymbol{d}} \Pi^{\tau}(\boldsymbol{d})\right\}
$$

where $\boldsymbol{d}$ denotes the total deformation and solvent-volume flux degrees of freedoms. 


\section{STRUCTURAL STABILITY ANALYSIS OF PERIODIC HYDROGELS}

In this section, we focus on the structural stability analysis of hydrogels with infinitely periodic microstructures. The effective response of such periodic microstructures can usually be determined via computations on a suitable representative volume element (RVE). In the present work, we refer to the smallest possible periodic RVE as the unit-cell RVE and denote it with $\mathcal{D}_{0}$. Although the uniqueness of the solution at small strains over such a unit-cell RVE is guarantied by convexity, at large strains convexity is physically not suitable and hence the effective response of a periodic microstructure cannot always be determined via computations on a unit-cell RVE [25]. In particular, because of microscopic instabilities the periodicity of the material can change which requires computations to be carried out on the new RVE usually contained of several unit-cells.

In the present contribution, we study stability of an equilibrium under infinitesimally small perturbations using Bloch-Floquet theorem and observe if the uniqueness of the underlying system of equations is maintained $[26,27,28]$. We start with the classical energy-based definition of the stability criterion [29]

$$
\Delta \Pi^{\tau}\left(d^{\star}, \delta d\right):=\Pi^{\tau}\left(d^{\star}+\varepsilon \delta d\right)-\Pi^{\tau}\left(d^{\star}\right)>0
$$

which requires that any infinitesimal perturbations in the neighborhood of an equilibrium state $\boldsymbol{d}^{\star}$ raise the potential of the system. If we consider a Taylor expansion of (20) around $\boldsymbol{d}^{\star}$

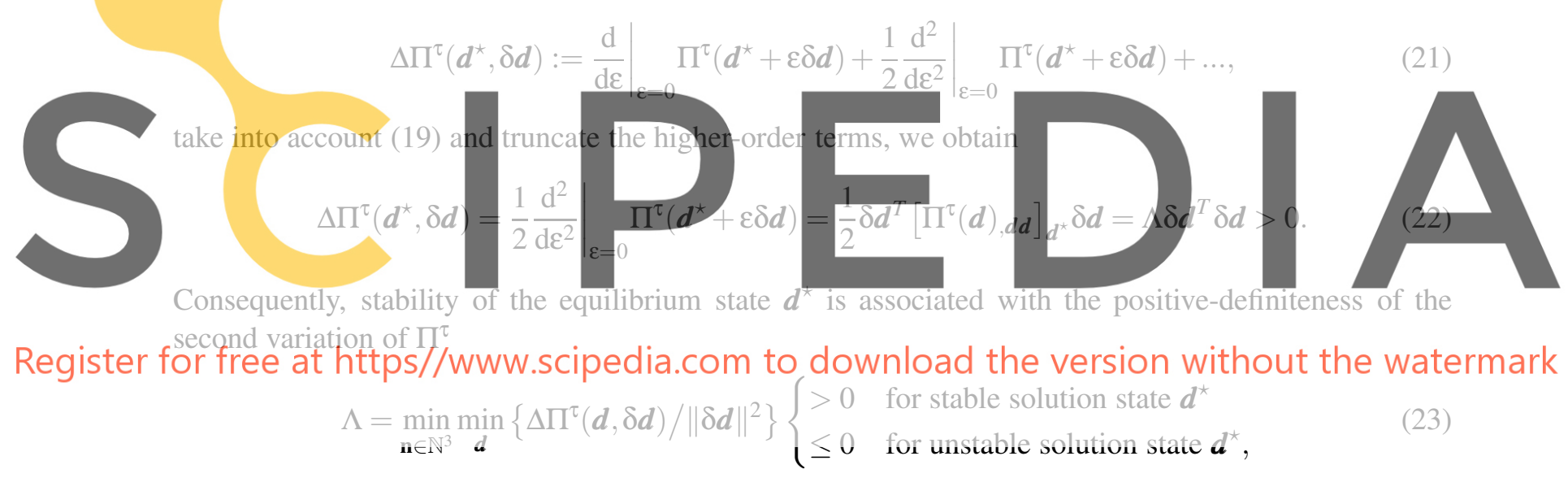

which needs to be checked for all possible RVEs constructed from $\mathbf{n} \in \mathbb{N}^{3}$ unit-cells in order to determine the critical instability point accurately. Since this requires significant effort, we use the Bloch-Floquet theory to determine the instabilities via computations over a unit-cell $\mathcal{D}_{0}$. Thus, instead of checking instabilities for various RVE sizes, we consider perturbations having various wavelengths, which can be expressed as follows

$$
\delta \boldsymbol{d}(\mathbf{X})=\delta \boldsymbol{d}_{\mathcal{D}_{0}}(\mathbf{X}) \exp [\mathbf{i k} \cdot \mathbf{X}] \quad \text { with } \quad \delta \boldsymbol{d}_{\mathcal{D}_{0}}^{+}=\delta \boldsymbol{d}_{\mathcal{D}_{0}}^{-} \quad \text { on } \quad \partial \mathcal{D}_{0}=\partial \mathcal{D}_{0}^{+} \cup \mathcal{D}_{0}^{-},
$$

where $\mathbf{k}$ is the Bloch vector and characterizes the wavelength of perturbations. As a result, we can modify the stabiltiy criterion (23) and obtain the final form as follows

$$
\Lambda=\min _{k_{i} \in[0, \pi]} \min _{\boldsymbol{d}}\left\{\Delta \Pi^{\tau}(\boldsymbol{d}, \delta \boldsymbol{d}) /\|\delta \boldsymbol{d}\|^{2}\right\} \begin{cases}>0 & \text { for stable solution state } \boldsymbol{d}^{\star} \\ \leq 0 & \text { for unstable solution state } \boldsymbol{d}^{\star}\end{cases}
$$


with the boundary conditions $\delta \boldsymbol{d}\left(\mathbf{X}^{+}\right)=\delta \boldsymbol{d}\left(\mathbf{X}^{-}\right) \exp \left[\mathbf{i k} \cdot\left(\mathbf{X}^{+}-\mathbf{X}^{-}\right)\right]$on the unit-cell RVE $\partial \mathcal{D}_{0}$. Depending on the critical Bloch vector components at an instability point, we differentiate local unit-cell periodic $\left(k_{i}=0\right)$, short-wavelength $\left(k_{i} \neq 0\right)$ and long-wavelength $\left(k_{i} \rightarrow 0\right)$ instabilities. The latter is usually associated with material instabilities of a corresponding homogeneous effective material [26].

\section{COMPUTATIONAL STUDY OF PATTERN-TRANSFORMING INSTABILITIES}

In this section, we investigate the pattern transformation of microstructures of periodic hydrogels as a result of microscopic instabilities. Our numerical studies focus on two-dimensional perforated microstructures with circular voids $\mathcal{H}_{0}$ of different volume fractions, see Fig. 2.

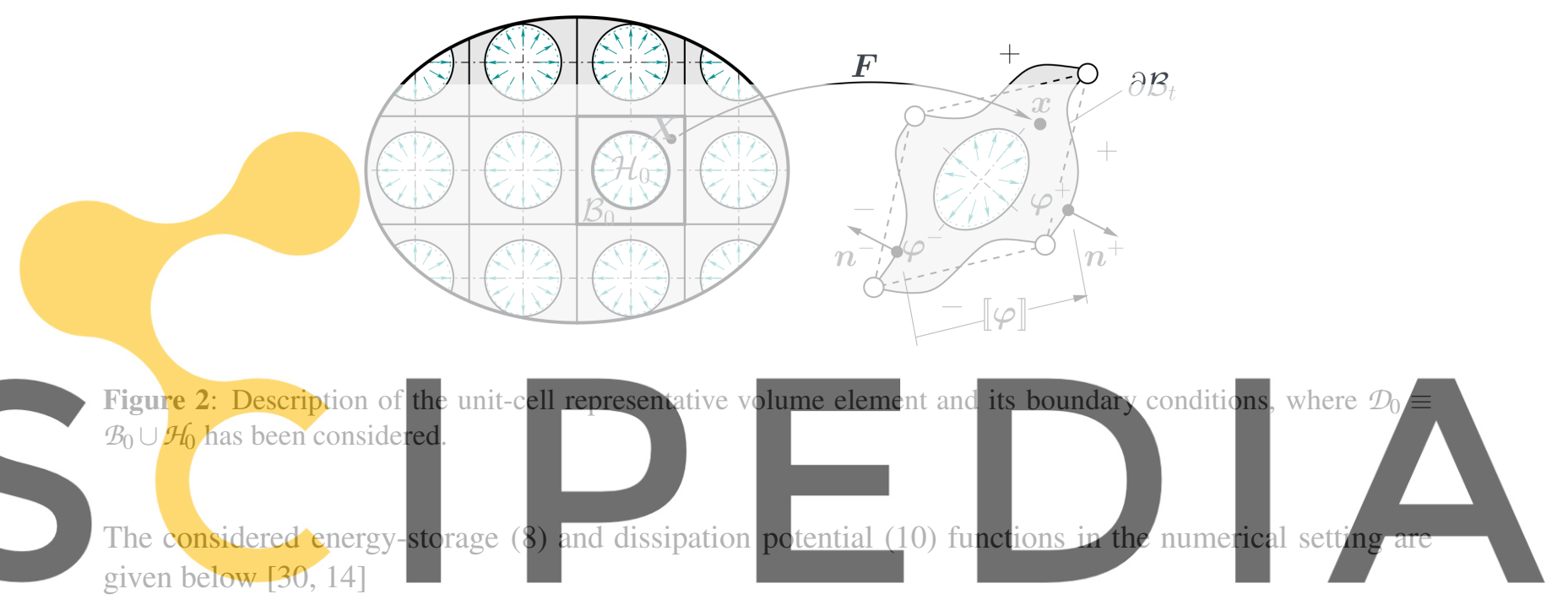

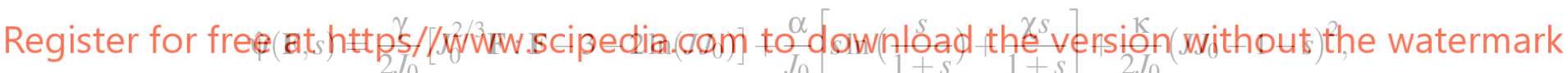

$$
\hat{\phi}\left(\mathbb{H} ; \mathbf{C}_{n}, s_{n}\right)=\frac{1}{2 J_{0}^{1 / 3} M s_{n}} \mathbf{C}_{n}:(\mathbb{H} \otimes \mathbb{H}) .
$$

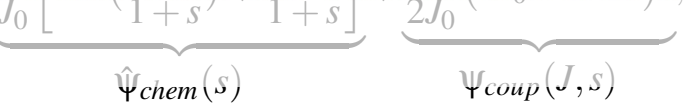

Since $(26)_{1}$ is singular at the dry state $s=0$, a stress-free swollen state of a hydrogel has been chosen

Table 1: Material parameters of perforated hydrogels

\begin{tabular}{ll}
\hline Parameters & Values \\
\hline Shear modulus, $\gamma /\left[\mathrm{N} / \mathrm{mm}^{2}\right]$ & 0.1 \\
Mixing modulus, $\alpha /\left[\mathrm{N} / \mathrm{mm}^{2}\right]$ & 40.0 \\
Flory-Huggins parameter, $\chi$ & 0.1 \\
Mobility parameter, $M /\left[\mathrm{mm}^{4} / \mathrm{N} \cdot \mathrm{s}\right]$ & $10^{-4}$ \\
Pre-swelling Jacobian, $J_{0}$ & 1.01 \\
Penalty parameter, $\kappa / \gamma$ & 10.0 \\
\hline
\end{tabular}



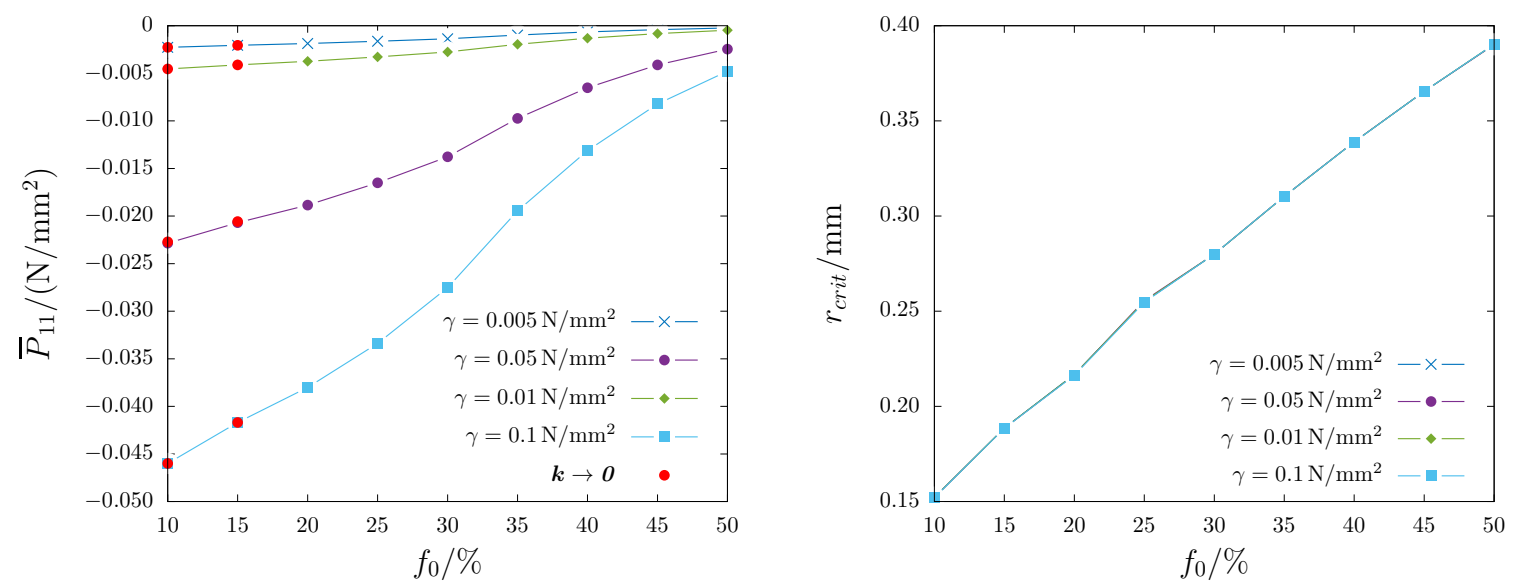

Figure 3: Influence of the shear modulus of hydrogel on the onset of instabilities (left) and corresponding critical deformed radius of the void (right) versus volume fraction of voids $f_{0}$.
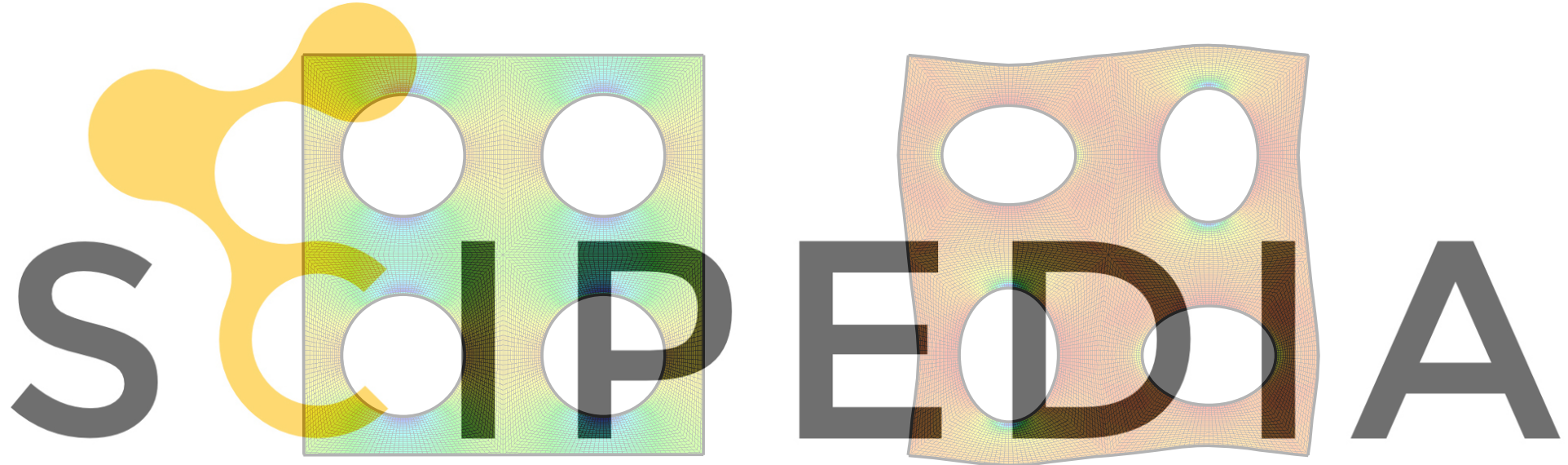

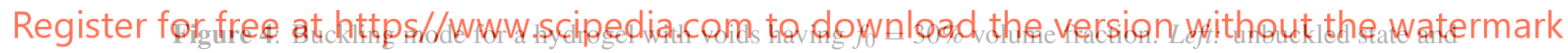
right: buckled state.

as the reference state which is characterized by the pre-swelling parameter $J_{0}$ and initial condition $s(t=$ $0)=s_{0}$, see, for example, $[19,20,21,14]$. The description of material parameters of the model as well as their values are provided in Table 1.

As we consider periodic microstructures, we apply periodic boundary conditions for the fluctuations of the deformation field and Dirichlet boundary conditions for the fluctuations of the solvent-volume flux on the outer boundary of the RVE, see [31]

$$
\llbracket \boldsymbol{\phi} \rrbracket=\overline{\mathbf{F}}(t) \cdot \llbracket \mathbf{X} \rrbracket \quad \text { and } \quad \llbracket \mathbb{H} \rrbracket=\mathbf{0} \quad \text { on } \quad \partial \mathcal{B}_{0},
$$

where $\overline{\mathbf{F}}=\frac{1}{\left|\mathcal{D}_{0}\right|} \int_{\mathcal{D}_{0}} \mathbf{F} \mathrm{d} V$ is the applied effective deformation gradient with $\mathcal{D}_{0}=\mathcal{B}_{0} \cup \mathcal{H}_{0} ; \llbracket(\cdot) \rrbracket$ denotes jump of a variable $(\cdot)$ across the RVE boundary, see Fig. 2. In the present contribution, we choose $\overline{\mathbf{F}}=\mathbf{1}$. On the inner boundary $\partial \mathcal{H}_{0}$ of the RVE, we apply the following traction and chemical potential boundary 
conditions

$$
\mathbf{t}=\mathbf{0} \quad \text { and } \quad \mu=\mu_{N}(t) \quad \text { on } \quad \partial \mathcal{H}_{0} .
$$

where the prescribed chemical potential $\mu_{N}$ is increased from the chemical potential of pre-swollen state $\mu_{N}(t=0)=\mu_{0}$ to $\mu_{N}(t=1)=0$ within 1.0 second. The considered solvent loading condition via the surface of the void resembles the loading from experimental works, e.g., refer to [8].

In Fig. 3, we illustrate the critical effective first Piola-Kirchhoff stresses $\bar{P}_{11}$ as well as the deformed critical radius of a material point $\left(0, r_{\text {void }}=\sqrt{\left(f_{0}\left|\mathcal{D}_{0}\right|\right) / \pi}=\sqrt{f_{0} / \pi}\right)$ for various volume fractions $f_{0}$ of the voids. The origin of the coordinate sytem is considered at the center of the unit-cell RVE. The plots include the shear modulus of the hydrogels to be in the range $\gamma=\{0.005,0.01,0.05,0.1\} \mathrm{N} / \mathrm{mm}^{2}$. From this numerical example, we observed that the critical effective stresses are strongly dependent on the shear modulus. The ratio of the critical stresses between two hydrogels with fixed void volume fraction are found to differ by the ratio of shear moduli of these microstructures. This is the reason for the independence of the critical radius of the voids from the shear modulus of the hydrogels. In most cases in this example, we observe short-wavelength instabilities yielding pattern transformations which can be captured by an enlarged RVE contained of $2 \times 2$ unit-cells. In Fig. 4, we illustrate this buckling mode for a hydrogel microstructure with voids of $f_{0}=30 \%$ volume fraction. The material parameters of the problem have been given in Table 1. The observed diamond plate pattern is in agreement with the experiments [7, 8]. Furthermore, we detected long-wavelength instabilities $(\mathbf{k} \rightarrow \mathbf{0})$ when the voids have the volume fractions of $f_{0}=10 \%$ and $f_{0}=15 \%$.
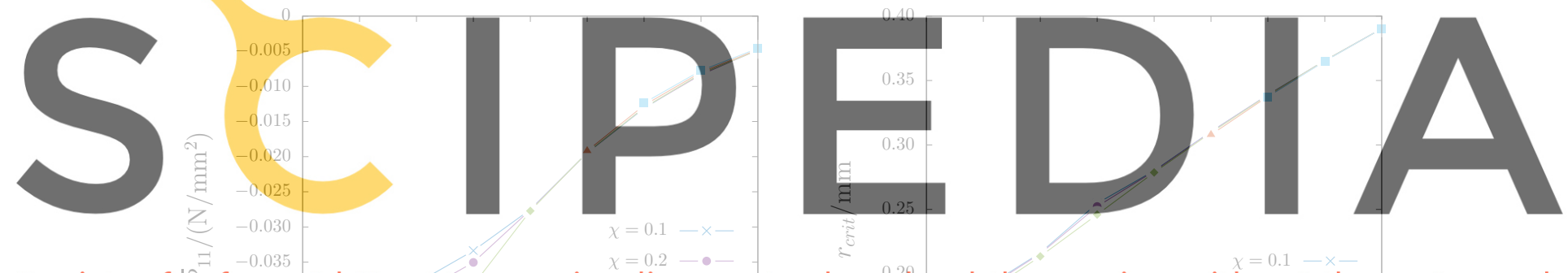

Register for free. at https//www.scipedia.com to downiload, the version without the watermark
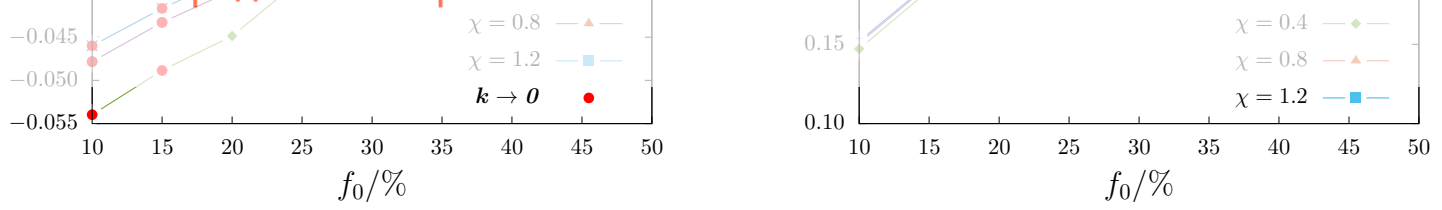

Figure 5: Influence of the Florry-Huggins parameter of hydrogel on the onset of instabilities (left) and corresponding critical deformed radius of the voids (right) versus volume fraction of voids $f_{0}$.

In Fig. 5, we also study the influence of the Flory-Huggings parameters $\chi$ on the onset of instabilities. The parameter is considered to be in the range $\chi=\{0.1,0.2,0.3,0.4,0.8,1.2\}$. The remaining material parameters can be found in Table 1. We observe that this parameter has a strong influence on the critical effective stresses. Nevertheless, we also observe marginal influence on the deformed critical radius of the void at the respective instability point. Similar to the previous case, we again detect mostly shortwavelength instabilities leading to the change of periodicity of the hydrogel microstructures. The new periodicity can be captured by an enlarged RVE of $2 \times 2$ unit-cells, see Fig. 4 . The long-wavelength 
instabilities $(\mathbf{k} \rightarrow \mathbf{0})$ correspond to the microstructures with voids having $f_{0}=10 \%$ and $f_{0}=15 \%$.

\section{SUMMARY}

The present work has investigated the development of instabilities in periodic hydrogels within a minimizationbased two-field formulation. The variational setting is implemented into a conforming finite-element formulation whereby we consider computational stability analysis using Bloch-Floquet theorem. In the current contribution, our studies considers under diffusion of the solvent through the surface of voids. The investigations account for various void volume fractions and shear moduli. Our results and observed pattern transformations are in good agreement with experimental data. In future studies we will consider the investigation of periodic hydrogels in two dimensions as well as in three dimensions.

\section{ACKNOWLEDGEMENT}

Funded by Deutsche Forschungsgemeinschaft (DFG, German Research Foundation) under Germany's Excellence Strategy - EXC 2075 - 390740016.

\section{REFERENCES}

[1] Enas M. Ahmed Hydrogel: Preparation, characterization, and applications: A review. J. Adv. Res. (2015) 2:(105-121)
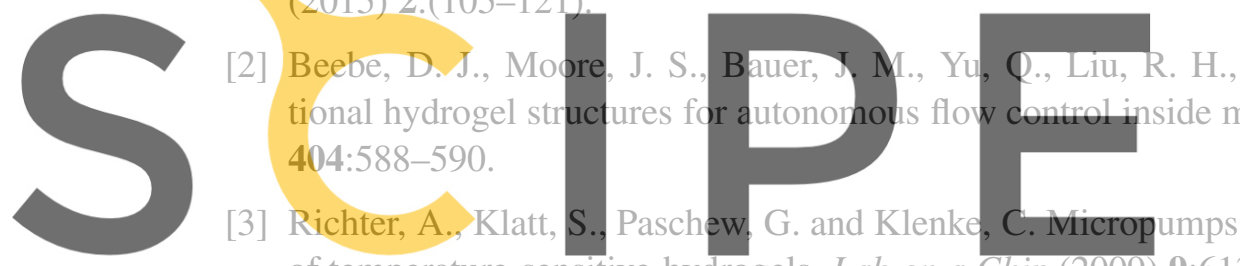
of temperature-sensitive hydrogels. Lab on a Chip (2009) 9:613-618.

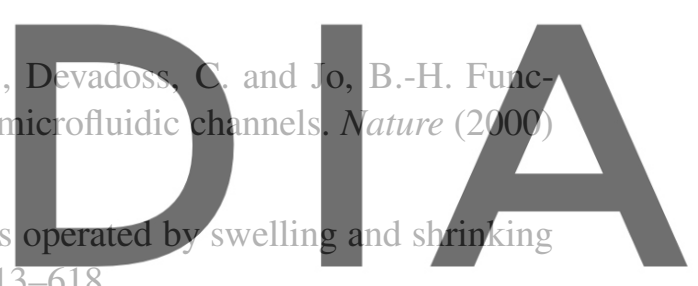

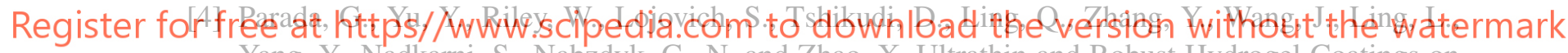
Yang, Y., Nadkarni, S., Nabzdyk, C., N. and Zhao, X. Ultrathin and Robust Hydrogel Coatings on Cardiovascular Medical Devices to Mitigate Thromboembolic and Infectious Complications. Adv. Healthc. Mater. (2020) 9:2001116(1-9).

[5] Wang, Z. J., Zhu, C. N., Hong, W., Wu, Z. L. and Zheng, Q. Cooperative deformations of periodically patterned hydrogels. Sci. $A d v$. (2017) 3:1-8.

[6] Zhang, H., Guo, X., Wu, J., Fang, D. and Zhang, Y. Soft mechanical metamaterials with unusual swelling behavior and tunable stress-strain curves. Sci. Adv. (2018) 4:1-10.

[7] Zhang, Y., Matsumoto, E. A., Peter, A., Lin, P.-C., Kamien, R. D. and Yang, S. One-step nanoscale assembly of complex structures via harnessing of an elastic instability. Nano Lett. (2008) 8:11921196.

[8] Zhu, X., Wu, G., Dong, R., Chen, C.-M. and Yang, S. Capillarity induced instability in responsive hydrogel membranes with periodic hole array. Soft Matter (2012) 8 (31):8088-8093.

[9] Dervaux, J. and Amar, M. B. Mechanical instabilities of gels. Annu. Rev. Condens. Matter Phys. (2012) 3:311-332. 
[10] Wu, G., Xia, Y. and Yang, S. Buckling, symmetry breaking, and cavitation in periodically microstructured hydrogel membranes. Soft Matter (2014) 10:1302-1399.

[11] Okumura, D., Kuwayama, T. and Ohno, N. Effect of geometrical imperfections on swelling-induced buckling patterns in gel films with a square lattice of holes. Int. J. Solids Struc. (2014) 51:154-163.

[12] Okumura, D., Inagaki, T. and Ohno, N. Effect of prestrains on swelling-induced buckling patterns in gel films with a square lattice of holes. Int. J. Solids Struc. (2015) 58:288-300.

[13] Miehe, C., Mauthe, S. and Teichtmeister, S. Minimization principles for the coupled problem of Darcy-Biot-type fluid transport in porous media linked to phase field modeling of fracture. J. Mech. Phys. Solids (2015) 82:186-217.

[14] Böger, L., Nateghi, A. and Miehe, C. Minimization-and Saddle-Point-Based Modeling of Diffusion-Deformation-Processes in Hydrogels. Int. J. Solids Struc. (2017) 121:257-274.

[15] Böger, L., Keip, M.-A. and Miehe, C. Minimization and saddle-point principles for the phase-field modeling of fracture in hydrogels. Comput. Mater. Sci. (2017) 138:474-485.

[16] Sriram, S., Polukhov, E. and Keip, M.-A. Transient stability analysis of composite hydrogel structures based on a minimization-type variational formulation. Int. J. Solids Struc. (2021) (submitted).

[17] Raviart, P.-A. and Thomas, J.-M. A mixed finite element method for 2-nd order elliptic problems. Mathematical aspects of finite element methods. Springer, (1977) p.292-315.

[18] Brezzi, F. and Fortin, M. Mixed and hybrid finite element methods. Springer-Verlag (1991).

[19] Hong, W., Zhao, X., Zhou, J. and Suo, Z. A theory of coupled diffusion and large deformation in polymeric gels. J. Mech. Phys. Solids (2008) 56 (5):1779-1793.

[20] Hong, W., Liu and Z., Suo, Z. Inhomogeneous swelling of a gel in equilibrium with a solvent and mechanical load. Int. J. Solids Struct. (2009) 46 (17):3282-3289.

[21] Chester, S. A. and Anand, L. A coupled theory of fluid permeation and large deformations for elastomeric materials. J. Mech. Phys. Solids (2010) 58 (11):1879-1906.

[22] Biot, M. A., [1941]. General theory of three-dimensional consolidation. J. Appl. Phys. 12 (2):155164.

[23] Coussy, O., Dormieux, L. and Detournay, E. From mixture theory to Biot's approach for porous media. Int. J. Solids Struct. (1998) 35 (34-35):4619-4635.

[24] Gurtin, M. E., Fried, E. and Anand, L. The mechanics and thermodynamics of continua. Cambridge University Press (2010).

[25] Müller, S. Homogenization of nonconvex integral functionals and cellular elastic materials. Arch. Ration. Mech. Anal. (1987) 99:189-212.

[26] Geymonat, G., Müller, S. and Triantafyllidis, N. Homogenization of nonlinearly elastic materials, microscopic bifurcation and macroscopic loss of rank-one convexity. Arch. Ration. Mech. Anal. (1993) 122:231-290.

[27] Polukhov, E., Vallicotti, D. and Keip, M.-A. Computational stability analysis of periodic electroactive polymer composites across scales. Comput. Method. Appl. Mech. Eng. (2018) 337:165-197. 
[28] Polukhov, E. and Keip, M.-A. Multiscale stability analysis of periodic magnetorheological elastomers. Mech. Mater. (2020), doi.org/10.1016/j.mechmat.2020.103699.

[29] Miehe, C., Schotte, J. and Schröder, J. Computational micro-macro transitions and overall moduli in the analysis of polycrystals at large strains. Comput. Mater. Sci. (1999) 16:372-382.

[30] Flory, P. J. and Rehner, J. J. Statistical mechanics of cross-linked polymer networks ii. swelling. J. Chem. Phys. (1943) 11 (11):521-526.

[31] Polukhov, E. and Keip, M.-A. Computational homogenization of transient chemo-mechanical processes based on a variational minimization principle. Adv. Model. Simul. Eng. Sci. (2020) 7:1-26. 